\title{
Pacific
}

Journal of

Mathematics

\section{A NOTE ON INTERMEDIATE SUBFACTORS}

\section{DIETMAR BISCH}

Volume 163 No. 2 


\section{A NOTE ON INTERMEDIATE SUBFACTORS}

\section{DieTMAR BISCH}

In this note we prove that if $N \subset M \subset P$ is an inclusion of $\mathrm{II}_{1}$ factors with finite Jones index such that $N \subset P$ has finite depth, then $N \subset M$ and $M \subset P$ have finite depth. We show this result by studying the iterated basic constructions for $M \subset P$ and $N \subset P$. In particular our proof gives detailed information about the graphs for $N \subset M$ resp. $M \subset P$. Furthermore, we give an abstract characterization of intermediate subfactors in terms of Jones projections in $N^{\prime} \cap P_{1}$, where $N \subset P \subset P_{1}$ is the basic construction for $N \subset P$ and give examples showing that if $N \subset M$ and $M \subset P$ have finite depth, then $N \subset P$ does not necessarily have finite depth.

1. Introduction. The problem of classifying subfactors of the hyperfinite $\mathrm{II}_{1}$ factor is one of the most challenging problems in operator algebras. Starting with an inclusion $N \subset M$ of hyperfinite $\mathrm{II}_{1}$ factors with finite Jones index $[M: N]<\infty$, one constructs the associated Jones tower of factors $N \subset M \subset M_{1} \subset M_{2} \subset \ldots$, where $M_{i+1}$ is the $\mathrm{II}_{1}$ factor obtained from the Jones basic construction for $M_{i-1} \subset M_{i}$ (see [Jo1]). The centralizer algebras $\left\{M_{i}^{\prime} \cap M_{j}\right\}_{i \leq j}$ are finite dimensional $C^{*}$-algebras sitting in the envelopping $\mathrm{II}_{1}$ factor $M_{\infty}={\widetilde{\cup M_{k}}}^{w}$. Furthermore, inclusions of four such algebras

$$
\begin{array}{ccccccc}
M_{i}^{\prime} & \cap & M_{k} & \subset & M_{i}^{\prime} & \cap & M_{k+1} \\
& \cup & & & & \cup & \\
M_{i+1}^{\prime} & \cap & M_{k} & \subset & M_{i+1}^{\prime} & \cap & M_{k+1}
\end{array}
$$

satisfy certain symmetry conditions: they form what is called a commuting square ([Po2], see also [GHJ]). All the information contained in this double sequence of finite dimensional algebras is actually contained in the following sequence of commuting squares

$$
\begin{array}{rlllllll}
M^{\prime} & \cap & M_{k} & \subset & M^{\prime} & \cap & M_{k+1} & \subset \cdots \\
& \cup & & & & \cup & & \\
M_{1}^{\prime} \cap M_{k} & \subset & M_{1}^{\prime} & \cap & M_{k+1} & \subset \cdots
\end{array}
$$

which is an invariant for the inclusion $N \subset M$, called the standard invariant ([Po4] or paragroup [Oc1]). From this sequence one can form the inclusion ${\overline{U_{k} M^{\prime} \cap M_{k}}}^{w} \subset \overline{\bigcup_{k} M_{1}^{\prime} \cap M_{k}}$ of hyperfinite $\mathrm{II}_{1}$ von Neumann algebras and ask if these algebras form a model for $N \subset M$, 
i.e. are (anti-)isomorphic to the inclusion $N \subset M$. Popa introduced recently a concept of amenability for inclusions $N \subset M$ ([Po3], [Po4]) and showed that precisely the amenable subfactors of $R$, the hyperfinite $\mathrm{II}_{1}$ factor, are classified by their standard invariant. A particular, but important class of amenable subfactors of $R$ are the finite depth subfactors, referring to the condition $\sup _{k} \operatorname{dim} Z\left(M^{\prime} \cap M_{k}\right)<\infty$, where $Z\left(M^{\prime} \cap M_{k}\right)$ denotes the center of $M^{\prime} \cap M_{k}$. Equivalently, this condition expresses the fact that the width of the Bratteli diagram describing the inclusions $\mathbb{C}=M^{\prime} \cap M \subset M^{\prime} \cap M_{1} \subset M^{\prime} \cap M_{2} \subset \ldots$ is bounded from a certain point on. Popa showed in ([Po2], see also [Oc1]) that finite depth subfactors $N$ of the hyperfinite $\mathrm{II}_{1}$ factor $M$ are classified by an initial commuting square

$$
\begin{array}{lllllll}
M^{\prime} & \cap & M_{k_{0}} & \subset & M^{\prime} & \cap & M_{k_{0}+1} \\
& \cup & & & & \cup & \\
M_{1}^{\prime} & \cap & M_{k_{0}} & \subset & M_{1}^{\prime} & \cap & M_{k_{0}+1}
\end{array}
$$

for $k_{0}$ large enough (which can be made precise). Subfactors of index $<4$ are automatically of finite depth and the associated commuting squares can be classified in terms of graphs of Coxeter-Dynkin type A, $\mathrm{D}, \mathrm{E}$ and certain connections on them ([B-N], [I1], [I2], [Jo1], [Ka], [Oc1], [Oc2], [SV]). Wenzl constructed interesting series of finite depth subfactors via braid group representations, generalizing Jones' original construction of subfactors of the hyperfinite $\mathrm{II}_{1}$ factor. It is by now well-known that Jones' discovery of certain remarkable braid group representations in the higher relative commutants of every finite index subfactor lead him to the construction of his link invariant, the Jones polynomial. Similarly, Wenzl's subfactors carry representations of the braid group in their higher relative commutants which can be used to obtain the HOMFLY and Kauffman polynomials using the same method as Jones' original construction of his link invariant ([Jo1], [Jo2], [We1], [We2]). The simplest finite depth subfactors are obtained by letting a finite group $G$ act by properly outer automorphisms of $R$ and considering the inclusion $R \subset R \rtimes G$. The canonical (classifying) commuting square of this inclusion contains all the information on $G$ and its representation theory: $G$ can be completely recovered from the inclusion. Similarly, if $H \subset G$ is a subgroup of $G$ of finite index, then $R \rtimes H \subset R \rtimes G$ is again a finite depth inclusion and the associated canonical commuting square can be described explicitly in terms of induced representations (for details of all this and more examples coming from groups, see [Bi2], [KY]). 
It is a well-known theorem in the theory of extensions of von Neumann algebras that if $G$ is a countable discrete group of outer automorphisms on the $\mathrm{II}_{1}$ factor $N$ and $P$ is a subfactor with $N \subset$ $P \subset N \rtimes G$, then there is a subgroup $H \subset G$ such that $P=N \rtimes H$ ([NT], [Su]). This result is quite apparent for a finite group $G$ and it is natural to ask if a similar result holds for finite depth subfactors, where the role of the group is played by the more general object, the canonical commuting square or the paragroup. In other words, given an inclusion of $\mathrm{II}_{1}$ factors $N \subset M \subset P$ such that $N \subset P$ has finite depth, does this force the finite depth condition on $N \subset M$ and $M \subset P$ ? We prove that this statement is indeed true, more precisely we show the following theorem:

TheOREM. Let $N \subset M \subset P$ be an inclusion of $\mathrm{II}_{1}$ factors with $[P: N]<\infty$ and assume $N \subset P$ has finite depth. Then $N \subset M$ and $M \subset P$ have finite depth.

This theorem will follow from a detailed study of the basic construction for $N \subset P$ and $M \subset P$. We are able to describe the higher relative commutants of $M \subset P$ completely in terms of the higher relative commutants of the inclusion $N \subset P$. In particular we obtain information on the graphs for $N \subset M$ and $M \subset P$ and our proof provides an algorithm for computing these graphs from the graphs for $N \subset P$.

In $\S 2$ we collect for the convenience of the reader some facts about the basic construction, fix the notation and prove some useful lemmas. We proceed then with the proof of our theorem. Furthermore, we give some examples showing that the converse of our theorem does not hold: if $N \subset M$ and $M \subset P$ have finite depth, then $N \subset M$ need not have finite depth, in fact $N \subset M$ need not even be amenable in the sense of Popa ([Po4]).

In $\S 3$ we give an abstract characterization of intermediate subfactors $M$ of a given irreducible inclusion $N \subset P$ in terms of Jones projections in $N^{\prime} \cap P_{1}$, where $N \subset P \subset P_{1}$ is the basic construction for $N \subset P$. This allows us to recognize intermediate subfactors by looking at the projections in $N^{\prime} \cap P_{1}$ and reconstruct the subfactor from these projections. ${ }^{1}$

2. The proof of the theorem. Let $N \subset M$ be an inclusion of $\mathrm{II}_{1}$ factors with finite Jones index $[M: N]$. We denote by $L^{2}\left(M, \operatorname{tr}_{M}\right)$

\footnotetext{
${ }^{1}$ Y. Kawahigashi informed us that A. Ocneanu has a characterization of intermediate subfactors using his Fourier transform.
} 
the closure of $M$ in the Hilbert norm $\|x\|_{2}=\operatorname{tr}_{M}\left(x^{*} x\right)^{1 / 2}$ induced by the unique trace $\operatorname{tr}_{M}$ on $M$. Let $e_{N}^{M}: L^{2}\left(M, \operatorname{tr}_{M}\right) \rightarrow L^{2}\left(N, \operatorname{tr}_{N}\right)$ be the orthogonal projection and let $J_{M}: L^{2}\left(M, \operatorname{tr}_{M}\right) \rightarrow L^{2}\left(M, \operatorname{tr}_{M}\right)$ be the canonical conjugation defined by $J_{M}(x)=x^{*}, x \in M$ viewed as a vector in $L^{2}\left(M, \operatorname{tr}_{M}\right)$. The algebra $M_{1}=v N\left(M, e_{N}^{M}\right)=$ $\left\langle M, e_{N}^{M}\right\rangle$, i.e. the von Neumann algebra generated by $M$ and $e_{N}^{M}$ in $B\left(L^{2}\left(M, \operatorname{tr}_{M}\right)\right)$ is called the basic construction for $N \subset M$ ([Jo1]). We recall ([Jo1]):

(1) $e_{N}^{M} x e_{N}^{M}=E_{N}^{M}(x) e_{N}^{M}$, where $E_{N}^{M}: M \rightarrow N$ is the unique trace preserving conditional expectation from $M$ onto $N$.

(2) $N=\left\{e_{N}^{M}\right\}^{\prime} \cap M$.

(3) $e_{N}^{M}\left\langle M, e_{N}^{M}\right\rangle e_{N}^{M}=N e_{N}^{M} \simeq N$.

(4) $\left\langle M, e_{N}^{M}\right\rangle=J_{M} N^{\prime} J_{M}$.

(5) $\left[J_{M}, e_{N}^{M}\right]=0$.

(6) There is a unique trace $\operatorname{tr}_{M_{1}}$ on $M_{1}$ such that $\operatorname{tr}_{M_{1}}\left(x e_{N}^{M}\right)=$ $[M: N]^{-1} \operatorname{tr}_{M}(x)$ for all $x \in M$.

(7) $M={\overline{\operatorname{span} N e_{N}^{M} N}}^{w}$ ([PiPo1]).

It is easy to see that $N \subset M$ has finite depth iff $M \subset M_{1}$ has finite depth iff $\sup _{k} \operatorname{dim} Z\left(M^{\prime} \cap M_{k}\right)<\infty$ iff $\sup _{k} \operatorname{dim} Z\left(M^{\prime} \cap M_{2 k}\right)<\infty$ iff $\sup _{k} \operatorname{dim} Z\left(M^{\prime} \cap M_{2 k+1}\right)<\infty$ iff $\sup _{k} \operatorname{dim} Z\left(N^{\prime} \cap M_{2 k}\right)<\infty$ iff $\sup _{k} \operatorname{dim} Z\left(N^{\prime} \cap M_{2 k+1}\right)<\infty$ (see for instance [Po2] for a proof). We will use at various instances the following simple abstract characterization of the basic construction ([PiPo2]): Let $N \subset M$ be an inclusion of $\mathrm{II}_{1}$ factors with $[M: N]<\infty$ and let $P$ be a $\mathrm{II}_{1}$ factor containing $M$ and a projection $p$ such that $[p, N]=0$ and $E_{M}^{P}(p)=[P: M]^{-1} 1_{M}=[M: N]^{-1} 1_{M}$, then $P$ is the basic construction for $N \subset M$, i.e. there is an isomorphism from $M_{1}$ onto $P$ leaving $M$ pointwise fixed and carrying $e_{N}^{M}$ to $p$.

It will be useful for the proof of our theorem to study the basic construction for certain reduced algebras. This is done in the next few lemmas.

LemMA 2.1. Let $N \subset M$ be $\mathrm{II}_{1}$ factors, $[M: N]<\infty$. Let $p \in N$, $q \in N^{\prime} \cap M$ be projections and consider the inclusion $q p N p q \subset p q M p q$ with trace $\operatorname{tr}_{p q M p q}(q p x p q)=\operatorname{tr}_{M}(p)^{-1} \operatorname{tr}_{N}(q)^{-1} \operatorname{tr}_{M}(p q x p q), x \in M$. Then the unique trace preserving conditional expectation $E_{p q N p q}^{p q M p q}$ : $p q M p q \rightarrow p q N p q$ is given by

$$
E_{p q N p q}^{p q M p q}(p q x p q)=\operatorname{tr}_{M}(q)^{-1} p E_{N}^{M}(q x q) p q, \quad x \in M .
$$


Proof. The proof is a straightforward computation and left to the reader.

Recall that $N \subset M$ is called extremal iff $\left.\operatorname{tr}_{M}\right|_{N^{\prime} \cap M}=\left.\operatorname{tr}_{N^{\prime}}\right|_{N^{\prime} \cap M}$ ([PiPo1]), which happens for instance whenever $N \subset M$ has finite depth.

LEMMA 2.2. Let $N \subset M$ be an extremal inclusion of $\mathrm{II}_{1}$ factors, $[M: N]<\infty$ and let $N \subset M \subset M_{1}=\left\langle M, e_{N}^{M}\right\rangle$ be the basic construction. Suppose we have projections $p \in N^{\prime} \cap M$ and $q \in M^{\prime} \cap M_{1}$ with $\operatorname{tr}_{M}(p)=\operatorname{tr}_{M_{1}}(q)$ such that $\operatorname{tr}_{M_{1}}\left(p q e_{N}^{M}\right)=[M: N]^{-1} \operatorname{tr}_{M}(p)$. Then

$$
N p q \subset(p M p) q \subset p q M_{1} p q
$$

is the basic construction for the pair $(N p q \subset(p M p) q) \simeq(N p \subset p M p)$.

Proof. Note that

$$
[q p M p q: N p q]=\left[p q M_{1} p q: p q M p q\right]=\operatorname{tr}_{M}(p)^{2}[M: N]
$$

since $N \subset M$ is extremal ([PiPo1]).

Consider $e:=\operatorname{tr}_{M}(p)^{-1} p q e_{N}^{M} p q \in p q M_{1} p q$, then

$$
\begin{aligned}
\operatorname{tr}_{p q M_{1} p q}(e) & =\operatorname{tr}_{M}(p)^{-2} \operatorname{tr}_{M_{1}}(e)=\operatorname{tr}_{M}(p)^{-3} \operatorname{tr}_{M_{1}}\left(p q e_{N}^{M}\right) \\
& =\operatorname{tr}_{M}(p)^{-2}[M: N]^{-1}=\left[p q M_{1} p q: p q M p q\right]^{-1} .
\end{aligned}
$$

Let $m$ be the unique element in $M$ satisfying $m e_{N}^{M}=q e_{N}^{M}$, i.e. $m=[M: N] E_{M}^{M_{1}}\left(q e_{N}^{M}\right)$. Then $m \in N^{\prime} \cap M$. We have $e^{2}=$ $\operatorname{tr}_{M}(p)^{-2} E_{N}^{M}(p m) p q e_{N}^{M} p q=e$, since by hypothesis

$$
\begin{aligned}
{[M: N]^{-1} \operatorname{tr}_{M}(p) } & =\operatorname{tr}_{M_{1}}\left(p q e_{N}^{M}\right)=\operatorname{tr}_{M_{1}}\left(p m e_{N}^{M}\right) \\
& =\operatorname{tr}_{M_{1}}\left(E_{N}^{M}(p m) e_{N}^{M}\right)=E_{N}^{M}(p m)[M: N]^{-1},
\end{aligned}
$$

which implies $E_{N}^{M}(p m)=\operatorname{tr}_{M}(p)$. In order to see that (1) is indeed the basic construction we use the above-mentioned characterization of the basic construction ([PiPo1]). Since $e$ clearly commutes with $N p q$, we only need to check that

$$
E_{p q M p q}^{p q M_{1} p q}(e)=\operatorname{tr}_{M}(p)^{-1}[M: N]^{-1} p q .
$$

By Lemma 2.1 we know that

$$
\begin{aligned}
E_{p q M p q}^{p q M_{1} p q}(e) & =\operatorname{tr}_{M}(p)^{-2} p E_{M}^{M_{1}}\left(q e_{N}^{M} q\right) p q \\
& =\operatorname{tr}_{M}(p)^{-2} p m[M: N]^{-1} m^{*} p q \\
& =\operatorname{tr}_{M}(p)^{-2}[M: N]^{-1} p m m^{*} p q .
\end{aligned}
$$


Computing traces on both sides of (3) gives

$$
\operatorname{tr}_{M}(p)^{2}=\operatorname{tr}_{M_{1}}\left(p m m^{*} p q\right)=\|p m\|_{2}^{2} \operatorname{tr}_{M_{1}}(q)
$$

and hence

$$
\|p m-p\|_{2}^{2}=\|p m\|_{2}^{2}-\operatorname{tr}_{M}(p)=0,
$$

i.e. $p m=p$. Note that this implies in particular that $p q e_{N}^{M}=p e_{N}^{M}$ (the condition $p m=p$ is actually equivalent to the condition on the traces in the statement of the lemma). Thus

$$
E_{p q M p q}^{p q M_{1} p q}(e)=\operatorname{tr}_{M}(p)^{-1}[M: N]^{-1} p q,
$$

which completes the proof.

REMARK 2.3. Note that if $N \subset M$ is extremal, given a projection $p$ as in the lemma, we can always find a projection $q \in M^{\prime} \cap$ $M_{1}$ such that $\operatorname{tr}_{M_{1}}\left(p q e_{N}^{M}\right)=\operatorname{tr}_{M}(p)[M: N]^{-1}, \operatorname{tr}_{M_{1}}(q)=\operatorname{tr}_{M}(p)$. Namely, let $q:=J_{M} p J_{M} \in J_{M}\left(N^{\prime} \cap M\right) J_{M}=M^{\prime} \cap M_{1}$, where $J_{M}$ denotes as usual the canonical conjugation on $L^{2}\left(M, \operatorname{tr}_{M}\right)$. We have then clearly $\operatorname{tr}_{M_{1}}(q)=\operatorname{tr}_{M}(p)$ (extremality) and $\operatorname{tr}_{M_{1}}\left(p J_{M} p J_{M} e_{N}^{M}\right)=$ $[M: N]^{-1} \operatorname{tr}_{M}(p)$ since $p J_{M} p J_{M} e_{N}^{M}=p e_{N}^{M}$.

The proof of the following lemma is trivial.

Lemma 2.4. Let $N \subset M$ be $\mathrm{II}_{1}$ factors, $[M: N]<\infty, p \in N a$ projection and $N \subset M \subset M_{1} \subset \ldots$ the basic construction. Then

$$
p N p \subset p M p \subset p M_{1} p \subset \ldots
$$

is that basic construction for $p N p \subset p M p$.

We describe now the construction which will be used to prove the theorem. Let

$$
M \subset P \subset Q_{1} \subset Q_{2} \subset \ldots
$$

be the Jones tower of factors obtained by iterating the basic construction for $M \subset P$. Similarly, let

$$
N \subset P \subset P_{1} \subset P_{2} \subset \ldots
$$

be the tower for $N \subset P$. Note that $N \subset M \subset P \subset Q_{1} \subset P_{1} \subset$ $B\left(L^{2}\left(P, \operatorname{tr}_{P}\right)\right)$. Let $\tilde{Q}_{2}:=\left\langle P_{1}, e_{Q_{1}}^{P_{1}}\right\rangle$ be the basic construction for $Q_{1} \subset P_{1}$, then $P \subset Q_{1} \subset P_{1} \subset \tilde{Q}_{2} \subset P_{2} \subset B\left(L^{2}\left(P_{1}, \operatorname{tr}_{P_{1}}\right)\right)$. Continuing this construction we obtain

$$
N \subset M \subset P \subset Q_{1} \subset P_{1} \subset \tilde{Q}_{2} \subset P_{2} \subset \tilde{Q}_{3} \subset P_{3} \subset \ldots,
$$


where $\tilde{Q}_{i-1} \subset P_{i} \subset \tilde{Q}_{i}=\left\langle P_{i-1}, e_{\tilde{Q}_{i-1}}^{P_{i-1}}\right\rangle$ is the basic construction. If we set $\alpha:=[M: N], \beta:=[P: M]$, then $\alpha \beta=[P: N]$ and the indices of the various inclusions are indicated as follows

$$
N \subset^{\alpha} M \subset^{\beta} P \subset^{\beta} Q_{1} \subset^{\alpha} P_{1} \subset^{\alpha} \tilde{Q}_{2} \subset^{\beta} P_{2} \subset^{\beta} \tilde{Q}_{3} \subset \ldots,
$$

and

$$
\begin{aligned}
\ldots & \subset^{\alpha} \tilde{Q}_{2 k-2} \subset^{\beta} P_{2 k-2} \subset^{\beta} \tilde{Q}_{2 k-1} \subset^{\alpha} P_{2 k-1} \subset^{\alpha} \tilde{Q}_{2 k} \\
& \subset^{\beta} P_{2 k} \subset^{\beta} \tilde{Q}_{2 k+1} \subset^{\alpha} P_{2 k+1} \subset^{\alpha} \ldots
\end{aligned}
$$

We will denote the Jones' projections in the following way: for instance $e_{\tilde{Q}_{2 k-1}}^{P_{2 k-1}}$ denotes the Jones projection which implements the conditional expectation from $P_{2 k-1}$ onto $\tilde{Q}_{2 k-1}$.

The computation in the next lemma will be used in the theorem.

LEMMA 2.5. With the notation as above we have

(1) $E_{\tilde{Q}_{2 k+1}}^{P_{2 k+1}}\left(e_{P_{2 k-1}}^{P_{2 k}}\right)=\alpha^{-1} e_{\tilde{Q}_{2 k}}^{P_{2 k}}$ and hence $e_{\tilde{Q}_{2 k+1}}^{P_{2 k+1}} e_{P_{2 k-1}}^{P_{2 k}} e_{P_{2 k+1}}^{P_{2 k+1}}=\alpha^{-1} e_{\tilde{Q}_{2 k}}^{P_{2 k}} e_{P_{2 k}}^{P_{2 k+1}}$, $k \geq 1$.

(2) $E_{\tilde{Q}_{2 k}}^{P_{2 k}}\left(e_{P_{2 k-2}}^{P_{2 k-1}}\right)=\beta^{-1} e_{\tilde{Q}_{2 k-1}}^{P_{2 k-1}}$ and hence $e_{\tilde{Q}_{2 k}}^{P_{2 k}} e_{P_{2 k-2}}^{P_{2 k-1}} e_{P_{2 k-1}}^{P_{2 k}}=\beta^{-1} e_{\tilde{Q}_{2 k-1}}^{P_{2 k-1}} e_{2 k-1}^{P_{2 k}}$, $k \geq 1,\left(\tilde{Q}_{1}:=Q_{1}\right)$.

Proof. The proof of (1) and (2) are identical, so we prove only (1). Since $e_{\tilde{Q}_{2 k}}^{P_{2 k}} \in \tilde{Q}_{2 k+1}$, we have that

$$
e_{\tilde{Q}_{2 k}}^{P_{2 k}} E_{\tilde{Q}_{2 k+1}}^{P_{2 k+1}}\left(e_{P_{2 k-1}}^{P_{2 k}}\right)=E_{\tilde{Q}_{2 k+1}}^{P_{2 k+1}}\left(e_{\tilde{Q}_{2 k}}^{P_{2 k}} e_{P_{2 k-1}}^{P_{2 k}}\right)=E_{\tilde{Q}_{2 k+1}}^{P_{2 k+1}}\left(e_{P_{2 k-1}}^{P_{2 k}}\right) .
$$

By [PiPo1] we know that there is a unique element $m \in P_{2 k}$ such that $E_{\tilde{Q}_{2 k+1}}^{P_{2 k+1}}\left(e_{P_{2 k-1}}^{P_{2 k}}\right)=E_{\tilde{Q}_{2 k+1}}^{P_{2 k+1}}\left(e_{P_{2 k-1}}^{P_{2 k}}\right) e_{\tilde{Q}_{2 k}}^{P_{2 k}}=m e_{\tilde{Q}_{2 k}}^{P_{2 k}}$. Applying $E_{P_{2 k}}^{\tilde{Q}_{2 k+1}}$ to both sides of the equation gives

$$
m=\operatorname{tr}_{P_{2 k+1}}\left(e_{\tilde{Q}_{2 k}}^{P_{2 k}}\right)^{-1} E_{P_{2 k}}^{P_{2 k+1}}\left(e_{P_{2 k-1}}^{P_{2 k}}\right)=\beta[P: N]^{-1} 1=\alpha^{-1} 1 .
$$

Since

$$
\begin{aligned}
e_{\tilde{Q}_{2 k+1}}^{P_{2 k+1}} e_{P_{2 k-1}}^{P_{2 k}} e_{P_{2 k}}^{P_{2 k+1}} & =e_{\tilde{Q}_{2 k+1}}^{P_{2 k+1}} e_{P_{2 k-1}}^{P_{2 k}} e_{\tilde{Q}_{2 k+1}}^{P_{2 k+1}} e_{P_{2 k}}^{P_{2 k+1}} \\
& =E_{\tilde{Q}_{2 k+1}}^{P_{2 k+1}}\left(e_{P_{2 k-1}}^{P_{2 k}}\right) e_{P_{2 k}}^{P_{2 k+1}},
\end{aligned}
$$

the second part also follows.

We restate now the theorem and give then the proof. 
THEOREM 2.6. Let $N \subset M \subset P$ be an inclusion of $\mathrm{II}_{1}$ factors with $[P: N]<\infty$ and assume $N \subset P$ has finite depth. Then $N \subset M$ and $M \subset P$ have finite depth.

Proof. (a) We show first that $M \subset P$ has finite depth. We actually prove the following statement by induction:

$$
\begin{aligned}
& \left(P \subset Q_{2} \subset Q_{4} \subset \cdots \subset Q_{2 k}\right) \\
& \quad \simeq\left(P f_{k-1} \subset f_{k-1} P_{2} f_{k-1} \subset \cdots \subset f_{k-1} P_{2 k} f_{k-1}\right),
\end{aligned}
$$

where $f_{k}:=e_{Q_{1}}^{P_{1}} e_{\tilde{Q}_{3}}^{P_{3}} \cdots e_{\tilde{Q}_{2 k+1}}^{P_{2 k+1}}$ (note that $f_{k}$ is a projection since all the $e_{\tilde{Q}_{r}}^{P_{r}}$ 's commute). First we show

$$
\left(P \subset Q_{1} \subset Q_{2}\right) \simeq\left(P \simeq P e_{Q_{1}}^{P_{1}} \subset e_{Q_{1}}^{P_{1}} \tilde{Q}_{2} e_{Q_{1}}^{P_{1}} \subset e_{Q_{1}}^{P_{1}} P_{2} e_{Q_{1}}^{P_{1}}\right) .
$$

Since $\tilde{Q}_{2}=\left\langle P_{1}, e_{Q_{1}}^{P_{1}}\right\rangle$ we have $e_{Q_{1}}^{P_{1}} \tilde{Q}_{2} e_{Q_{1}}^{P_{1}}=Q_{1} e_{Q_{1}}^{P_{1}}$ and therefore $(P \subset$ $\left.Q_{1}\right) \simeq\left(P e_{Q_{1}}^{P_{1}} \subset Q_{1} e_{Q_{1}}^{P_{1}}\right)$ and hence

$$
\begin{aligned}
\left(P \subset Q_{1} \subset Q_{2}\right) & \simeq\left(P e_{Q_{1}}^{P_{1}} \subset Q_{1} e_{Q_{1}}^{P_{1}} \subset\left\langle Q_{1} e_{Q_{1}}^{P_{1}}, P e_{Q_{1}}^{P_{1}}\right\rangle\right) \\
& \simeq\left(P e_{Q_{1}}^{P_{1}} \subset e_{Q_{1}}^{P_{1}} \tilde{Q}_{2} e_{Q_{1}}^{P_{1}} \subset e_{Q_{1}}^{P_{1}} P_{2} e_{Q_{1}}^{P_{1}}\right),
\end{aligned}
$$

where the last isomorphism is checked by using again the abstract characterization of the basic construction ([PiPo2]): Set $e:=e_{Q_{1}}^{P_{1}} e_{P}^{P_{1}} e_{Q_{1}}^{P_{1}}$ and note that actually $e_{Q_{1}}^{P_{1}} e_{P}^{P_{1}}=e_{P}^{P_{1}}$. Thus $e$ is a projection in $e_{Q_{1}}^{P_{1}} P_{2} e_{Q_{1}}^{P_{1}}$ of the right trace, namely $\beta$. Clearly $\left[e, P e_{Q_{1}}^{P_{1}}\right]=0$ and it remains to show that

$$
E_{Q_{Q_{1}}^{P_{1}} \tilde{Q}_{2} e_{Q_{1}}^{P_{1}}}^{e_{Q_{1}}^{P_{1}} P_{2}^{P_{1}} e^{P_{1}}}(e)=\beta^{-1} e_{Q_{1}}^{P_{1}}
$$

To simplify the notation we set $A:=e_{Q_{1}}^{P_{1}} P_{2} e_{Q_{1}}^{P_{1}}$ and we recall that $\tilde{Q}_{2}={\overline{\operatorname{span} P_{1} e_{Q_{1}}^{P_{1}} P_{1}}}^{w}$. We need to check that

$$
\operatorname{tr}_{A}\left(e_{Q_{1}}^{P_{1}} x e_{Q_{1}}^{P_{1}} e\right)=\beta^{-1} \operatorname{tr}_{A}\left(e_{Q_{1}}^{P_{1}} x e_{Q_{1}}^{P_{1}}\right),
$$

for all $x \in \tilde{Q}_{2}$. Let $y \in Q_{1}$ with $e_{Q_{1}}^{P_{1}} x e_{Q_{1}}^{P_{1}}=y e_{Q_{1}}^{P_{1}}$, then

$$
\begin{aligned}
\operatorname{tr}_{A}\left(e_{Q_{1}}^{P_{1}} x e_{Q_{1}}^{P_{1}} e_{P}^{P_{1}} e_{Q_{1}}^{P_{1}}\right) & =\left[P_{1}: Q_{1}\right] \operatorname{tr}_{P_{2}}\left(y e_{Q_{1}}^{P_{1}} e_{P}^{P_{1}}\right)=\alpha \operatorname{tr}_{P_{2}}\left(y e_{P}^{P_{1}}\right) \\
& =\left[P_{1}: P\right]^{-1} \alpha \operatorname{tr}_{P_{2}}(y)=(\beta)^{-1} \alpha \operatorname{tr}_{P_{2}}\left(y e_{Q_{1}}^{P_{1}}\right) \\
& =\beta^{-1} \operatorname{tr}_{A}\left(e_{Q_{1}}^{P_{1}} x e_{Q_{1}}^{P_{1}}\right) .
\end{aligned}
$$


This proves (12). In particular we have $\left(P \subset Q_{2}\right) \simeq\left(P e_{Q_{1}}^{P_{1}} \subset e_{Q_{1}}^{P_{1}} P_{2} e_{Q_{1}}^{P_{1}}\right)$ (note that this implies already that $P \subset Q_{2}$ and hence $M \subset P$ have finite depth since $P \subset P_{2}$ does (assuming we know that reduced subfactors of finite depth subfactors have finite depth). Since we want to get an explicit description of the higher relative commutants, we want to prove more, namely (9)) .

For clarity of exposition let us also do the next step of the induction. Since $P \subset P_{2} \subset P_{4}$ is the basic construction, $e_{Q_{1}}^{P_{1}} \in P^{\prime} \cap P_{2}, e_{\tilde{Q}_{3}}^{P_{3}} \in$ $P_{2}^{\prime} \cap P_{4}, \operatorname{tr}_{P_{2}}\left(e_{Q_{1}}^{P_{1}}\right)=\operatorname{tr}_{P_{4}}\left(e_{\tilde{Q}_{3}}^{P_{3}}\right)=\alpha^{-1}$, we only need to check

$$
\operatorname{tr}_{P_{4}}\left(e_{Q_{1}}^{P_{1}} e_{\tilde{Q}_{3}}^{P_{3}} e_{P}^{P_{2}}\right)=\left[P_{2}: P\right]^{-1} \operatorname{tr}_{P_{2}}\left(e_{Q_{1}}^{P_{1}}\right)=[P: N]^{-2} \alpha^{-1}
$$

in order to be able to apply Lemma 2.2. By [PiPo2] we know that $e_{P}^{P_{2}}=[P: N] e_{P_{1}}^{P_{2}} e_{P}^{P_{1}} e_{P_{2}}^{P_{3}} e_{P_{1}}^{P_{2}}$ and hence

$$
\begin{aligned}
\operatorname{tr}_{P_{4}}\left(e_{Q_{1}}^{P_{1}} e_{\tilde{Q}_{3}}^{P_{3}} e_{P}^{P_{2}}\right) & =[P: N] \operatorname{tr}_{P_{4}}\left(e_{Q_{1}}^{P_{1}} e_{P_{1}}^{P_{2}} e_{P}^{P_{1}} e_{P_{2}}^{P_{3}} e_{P_{1}}^{P_{2}} e_{\tilde{Q}_{3}}^{P_{3}}\right) \\
& =[P: N] \alpha^{-1} \operatorname{tr}_{P_{4}}\left(e_{Q_{1}}^{P_{1}} e_{P_{1}}^{P_{2}} e_{P}^{P_{1}} e_{P_{2}}^{P_{3}} e_{\tilde{Q}_{2}}^{P_{2}}\right) \quad(\text { Lem } \\
& =\alpha^{-1} \operatorname{tr}_{P_{4}}\left(e_{Q_{1}}^{P_{1}} e_{P_{1}}^{P_{2}} e_{P}^{P_{1}} e_{\tilde{Q}_{2}}^{P_{2}}\right) \\
& =\alpha^{-1} \beta^{-1} \operatorname{tr}_{P_{4}}\left(e_{Q_{1}}^{P_{1}} e_{P_{1}}^{P_{2}} e_{Q_{1}}^{P_{1}}\right) \quad(\text { Lemma 2.5) } \\
& =[P: N]^{-2} \operatorname{tr}_{P_{4}}\left(e_{Q_{1}}^{P_{1}}\right)=[P: N]^{-2} \alpha^{-1} .
\end{aligned}
$$

Thus we can apply Lemma 2.2 to $\left(P \subset Q_{2}\right) \simeq\left(P e_{Q_{1}}^{P_{1}} \subset e_{Q_{1}}^{P_{1}} P_{2} e_{Q_{1}}^{P_{1}}\right)$ $\simeq\left(P e_{Q_{1}}^{P_{1}} e_{\tilde{Q}_{3}}^{P_{3}} \subset e_{Q_{1}}^{P_{1}} e_{\tilde{Q}_{3}}^{P_{3}} P_{2} e_{Q_{1}}^{P_{1}} e_{\hat{Q}_{3}}^{P_{3}}\right)$ and obtain

$$
\left(P \subset Q_{2} \subset Q_{4}\right) \simeq\left(P f_{1} \subset f_{1} P_{2} f_{1} \subset f_{1} P_{4} f_{1}\right) \text {. }
$$

This shows (9) for $k=1,2$. Now suppose (9) holds for $k$, and we will show it for $k+1$. To this end it is enough to show that

$$
\left(Q_{2 k-2} \subset Q_{2 k} \subset Q_{2 k+2}\right) \simeq\left(f_{k} P_{2 k-2} f_{k} \subset f_{k} P_{2 k} f_{k} \subset f_{k} P_{2 k+2} f_{k}\right) .
$$

Note that $f_{k-2} \in P_{2 k-2}$, thus by Lemma 2.4 (and [PiPo2]) we know that $f_{k-2} P_{2 k-2} f_{k-2} \subset f_{k-2} P_{2 k} f_{k-2} \subset f_{k-2} P_{2 k+2} f_{k-2}$ is the basic construction. We want to apply Lemma 2.2 with $N \leftrightarrow f_{k-2} P_{2 k-2} f_{k-2}$, $M \leftrightarrow f_{k-2} P_{2 k} f_{k-2}, M_{1} \leftrightarrow f_{k-2} P_{2 k+2} f_{k-2}, p \leftrightarrow f_{k-1}$ and $q \leftrightarrow$ $e_{\tilde{Q}_{2 k+1}}^{P_{2 k+1}} f_{k-2}$. Since $f_{k-2} P_{2 k-2} f_{k-2} \subset f_{k-2} P_{2 k} f_{k-2}$ is clearly extremal (because $P_{2 k-2} \subset P_{2 k}$ has finite depth) and $\operatorname{tr}_{f_{k-2} P_{2 k+2} f_{k-2}}\left(e_{\tilde{Q}_{2 k+1}}^{P_{2 k+1}} f_{k-2}\right)=$ 
$\operatorname{tr}_{f_{k-2} P_{2 k} f_{k-2}}\left(f_{k-1}\right)=\alpha^{-1}$, we only need to check the trace condition in Lemma 2.2, i.e.

$$
\operatorname{tr}_{f_{k-2} P_{2 k+2} f_{k-2}}\left(f_{k} e_{f_{k-2} P_{2 k-2} f_{k-2}}^{f_{k-2} f_{k-2}}\right)=\alpha^{-1}[P: N]^{-2} .
$$

But using [PiPo2] and Lemma 2.5 we compute

$$
\begin{aligned}
& \operatorname{tr}_{f_{k-2} P_{2 k+2} f_{k-2}}\left(f_{k} e_{f_{k-2} P_{2 k-2} f_{k-2} P_{k-2}}^{f_{k-1}}\right)=\alpha^{k-1} \operatorname{tr}_{P_{2 k+2}}\left(f_{k} e_{P_{2 k-2}}^{P_{2 k}}\right) \\
& =\alpha^{k-1}[P: N] \operatorname{tr}_{P_{2 k+2}}\left(f_{k} e_{P_{2 k-1}}^{P_{2 k}} e_{P_{2 k-2}}^{P_{2 k-1}} e_{P_{2 k+1}}^{P_{2 k+1}} e_{P_{2 k-1}}^{P_{2 k}}\right) \\
& =\alpha^{k-1}[P: N] \alpha^{-1} \operatorname{tr}_{P_{2 k+2}}\left(f_{k-1} e_{P_{2 k-1}}^{P_{2 k}} e_{P_{2 k-2}}^{P_{2 k-1}} e_{P_{2 k+1}}^{P_{2 k+1}} e_{\tilde{Q}_{2 k}}^{P_{2 k}}\right) \\
& =\alpha^{k-2} \operatorname{tr}_{P_{2 k+2}}\left(f_{k-1} e_{P_{2 k-1}}^{P_{2 k}} e_{P_{2 k-2}}^{P_{2 k-1}} e_{\tilde{Q}_{2 k}}^{P_{2 k}}\right) \\
& =\alpha^{k-1} \alpha^{-1} \beta^{-1} \operatorname{tr}_{P_{2 k+2}}\left(f_{k-1} e_{P_{2 k-1}}^{P_{2 k}}\right) \\
& =[P: N]^{-2} \alpha^{k-1} \operatorname{tr}_{P_{2 k}}\left(f_{k-1}\right)=[P: N]^{-2} \alpha^{-1} .
\end{aligned}
$$

Applying Lemma 2.2 gives (14) and completes the induction, i.e. (9) holds. Therefore we proved that

$$
P^{\prime} \cap Q_{2 r} \simeq f_{k-1}\left(P^{\prime} \cap P_{2 r}\right) f_{k-1}, \quad 1 \leq r \leq k,
$$

which implies that $P \subset Q_{2}$ and hence $M \subset P$ have finite depth since $P \subset P_{2}$ does by assumption $\left(P \subset P_{2}\right.$ has finite depth iff $N \subset P$ has finite depth, [Po2]).

(b) The fact that $N \subset M$ has finite depth follows now from a simple duality argument. We can choose $P_{-1}, M_{-1}$ such that $P_{-1} \subset$ $M_{-1} \subset N \subset M \subset P$ and $P_{-1} \subset N \subset P$ and $M_{-1} \subset N \subset M$ are basic constructions. $P_{-1} \subset N$ has finite depth since $N \subset P$ does by hypothesis and hence $M_{-1} \subset N$ has finite depth by what we just proved. But $M_{-1} \subset N$ has finite depth iff $N \subset M$ has finite depth, which completes the proof of the theorem.

The main motivation for giving a detailed proof of the theorem is the fact that we want to obtain information on the principal graphs (see [GHJ] for terminology) for $N \subset M$ and $M \subset P$ in terms of the principal graphs for $N \subset P$. Some information can indeed be obtained by looking at the Bratteli diagrams of the inclusions of higher relative commutants associated to $N \subset P$. We summarize in the next corollary what can be read off the above proof.

Corollary 2.7. Let $N \subset M \subset P$ be as in the theorem. Then the Bratteli diagram of $N \subset M$ (from 2 to 2 steps) is obtained as a 
subdiagram of the Bratteli diagram for $N \subset P$ (from 2 to 2 steps). Similarly, the Bratteli diagram for $P \subset Q_{1}$ (from 2 to 2 steps), which is the "dual" Bratteli diagram for $M \subset P$, is obtained as a subdiagram of the Bratteli diagram for $P \subset P_{1}$ (from 2 to 2 steps), which is the "dual" Bratteli diagram for $N \subset P$ (from 2 to 2 steps). Furthermore, the method in the above proof gives an explicit algorithm to compute these Bratteli diagrams (see (9), (16)).

Note that the corollary generalizes what happens in the situation $N \subset N \rtimes H \subset N \rtimes G$, where $H \subset G$ are finite groups and $H$ is a subgroup of finite index of $G$ (see for instance [KY]). Even for these subfactors it is impossible to find a general and more explicit relation between the principal graphs of the "big" inclusion and the ones of the two "smaller" inclusions.

Let us also remark that since finite depth subfactors are classified by their canonical commuting squares or paragroups ([Po1], [Oc1]), our theorem can be viewed as defining a quotient of the canonical paragroup associated to $N \subset P$ by the one associated to $N \subset M$ : the result is again a paragroup, namely the one associated to $M \subset P$. As pointed out previously by Ocneanu, the quotient $G / H$ of two groups (viewed as paragroups) $H \subset G$ with $[G: H]<\infty$ is always a paragroup. We intend to explore these ideas further in a future paper.

We mention that Popa has shown independently the analogous statement of the theorem with "finite depth subfactor" replaced by "amenable subfactor", which does not imply our theorem.

Finally we give some examples of finite depth subfactors $N \subset M$, $M \subset P$ such that $N \subset P$ is not of finite depth and/or amenable in the sense of Popa ([Po3], [Po4]). Let $N$ be the hyperfinite $\mathrm{II}_{1}$ factor and consider $N \subset N \rtimes_{\alpha} \mathbb{Z}_{2} \subset\left(N \rtimes_{\alpha} \mathbb{Z}_{2}\right) \rtimes_{\beta} \mathbb{Z}_{2}=: P$, with $\alpha$ and $\beta$ outer actions of $\mathbb{Z}_{2}$ on $N$ such that period $(\alpha \beta)=\infty$. Then $N \subset P$ has standard graph $D_{\infty}$, i.e. is not of finite depth ([Po3]). Haagerup showed in [Ha] that if there are subfactors $N \subset M \subset P$ of the hyperfinite $\mathrm{II}_{1}$ factor where $N \subset M$ and $M \subset P$ have index 2 resp. $4 \cos ^{2} \pi / 5$ (hence are of finite depth), then $N \subset P$ cannot be amenable. Another such example was mentioned to us by V.F.R. Jones: take $\operatorname{PSL}(2, \mathbb{Z})=\mathbb{Z}_{2} * \mathbb{Z}_{3}$ with generators $\alpha$ and $\beta$. Let $\alpha$ and $\beta$ act on the hyperfinite $\mathrm{II}_{1}$ factor by properly outer automorphisms such that the action of $\operatorname{PSL}(2, \mathbb{Z})$ is ergodic on central sequences ([Jo3]) and consider the inclusion $N^{\alpha} \subset N \subset N \rtimes_{\beta} \mathbb{Z}_{3}$ of index $2 \cdot 3=$ 6 , which cannot be amenable since all the central sequences for $P$ contained in the subfactor are trivial ([Bi1]). 
Given an inclusion of $\mathrm{II}_{1}$ factors $N \subset P,[P: N]<\infty$, we would like to determine all intermediate subfactors of $N \subset P$ from looking only at this given inclusion. This can indeed be done and we settle this problem in the following section.

3. Abstract characterzation of intermediate subfactors. Consider $\mathrm{II}_{1}$ factors $N \subset P,[P: N]<\infty$, not necessarily of finite depth and let $N \subset P \subset P_{1}$ be the basic construction. If there is an intermediate subfactor $N \subset M \subset P$, then the Jones' projection $e_{M}^{P} \in N^{\prime} \cap P_{1}$ can be abstractly characterized among the projections in $N^{\prime} \cap P_{1}$. Let us first collect the properties of $e_{M}^{P}$.

Proposition 3.1. Let $N \subset M \subset P$ be $\mathrm{II}_{1}$ factors with $[P: N]<$ $\infty$. Let $N_{1} \subset N \subset P \subset P_{1}, M_{-1} \subset N \subset M$ and $M \subset P \subset Q_{1}$ be basic constructions with $N \subset M \subset P \subset Q_{1} \subset P_{1}$ and $N_{1} \subset M_{-1} \subset$ $N \subset M \subset P$. Then

(1) $e_{M}^{P} \in N^{\prime} \cap P_{1}$.

(2) $e_{M}^{P} e_{N}^{P}=e_{N}^{P}$.

(3) $E_{P}^{P_{1}}\left(e_{M}^{P}\right)=[P: M]^{-1} 1_{P}$.

(4) $e_{M}^{P} P e_{M}^{P}=M e_{M}^{P} \subset P e_{M}^{P}$.

(5) $e_{M}^{P} e_{N_{1}}^{N} e_{N}^{P}=E_{M}^{P}\left(e_{N_{1}}^{N}\right) e_{N}^{P}$ and $E_{M}^{P}\left(e_{N_{1}}^{N}\right)=[P: M]^{-1} e_{M_{-1}}^{N}$.

Proof. (1)-(4) follow from properties of Jones projections, (5) is proved using the method of Lemma 2.5.

It will turn out that (1)-(5) characterize the Jones projections coming from intermediate subfactors, but that actually not all these properties are needed to give this characterization.

Consider the basic construction $N \subset P \subset P_{1}$, choose a subfactor $N_{1} \subset N$ such that $N_{1} \subset N \subset P$ is the basic construction and and define the set

$\operatorname{IS}(N, P):=\left\{q \in N^{\prime} \cap P_{1}\right.$ projection such that

(1) $q e_{N}^{P}=e_{N}^{P}$.

(2) $E_{P}^{P_{1}}(q) \in \mathbb{C}$.

(3) Let $m$ be the unique element in $P$ satisfying $q e_{N_{1}}^{N} e_{N}^{P}=m e_{N}^{P}$, i.e. $m=E_{P}^{P_{1}}\left(q e_{N_{1}}^{N} e_{N}^{P}\right)[P: N]$.

Assume that $m$ is a scalar multiple of a projection.\}

Note that (2) is equivalent to requiring that $E_{P}^{P_{1}}(q)=\operatorname{tr}_{P_{1}}(q) 1_{P}$ and that (3) does not depend on the choice of the subfactor $N_{1} \subset N$ 
(two different choices are conjugate by a unitary in $N$ ). Furthermore, it is clear that we always have $1, e_{N}^{P} \in \operatorname{IS}(N, P)$ and that every Jones projection $e_{M}^{P}$ coming from an intermediate subfactor $M$ lies in $\operatorname{IS}(N, P)$. Conversely, we prove that any projection in $\operatorname{IS}(N, P)$ is a Jones projection coming from an intermediate subalgebra $N \subset$ $M \subset P$.

TheOREM 3.2. Let $N \subset P$ be $\mathrm{II}_{1}$ factors with $[P: N]<\infty$. Then every projection $q \in \mathrm{IS}(N, P)$ implements a conditional expectation from $P$ onto the intermediate subalgebra $M:=\{q\}^{\prime} \cap P$. If $M$ is $a$ factor, then its index $[P: M]$ in $P$ is equal to $\operatorname{tr}_{P_{1}}(q)^{-1}$. In particular, $q=1$ corresponds to the subfactor $P$ and $q=e_{N}^{P}$ to $N$.

Proof. Let $q \in \mathrm{IS}(N, P)$ and $m=E_{P}^{P_{1}}\left(q e_{N_{1}}^{N} e_{N}^{P}\right)[P: N]=\lambda p$ for some $\lambda \in \mathbb{C}$ and a projection $p \in P$. We show first that $q$ implements a conditional expectation from $P$ onto $M:=\{q\}^{\prime} \cap P$, a finite von Neumann algebra. Since $q P q=\overline{\operatorname{span} q N e_{N_{1}}^{N} N q}=\overline{\operatorname{span} N q e_{N_{1}}^{N} q N}$, we need to determine $q e_{N_{1}}^{N} q$. But

$$
q e_{N_{1}}^{N} q=[P: N] q e_{N_{1}}^{N} e_{N}^{P} e_{N_{1}}^{N} q=[P: N] \lambda^{2} p e_{N}^{P} p .
$$

We first compute $\lambda$ :

$$
\operatorname{tr}_{P}(p)=\lambda^{-1} \operatorname{tr}_{P_{1}}\left(q e_{N_{1}}^{N} e_{N}^{P}\right)[P: N]=\lambda^{-1}[P: N]^{-1} .
$$

Since $m e_{N_{1}}^{N}=[P: N] E_{P}^{P_{1}}\left(q e_{N_{1}}^{N} e_{N}^{P} e_{N_{1}}^{N}\right)=\operatorname{tr}_{P_{1}}(q) e_{N_{1}}^{N}$, we get that $p e_{N_{1}}^{N}=$ $e_{N_{1}}^{N} p$ is a projection. But $p e_{N_{1}}^{N}=\lambda^{-1} \operatorname{tr}_{P_{1}}(q) e_{N_{1}}^{N}$, thus $\lambda=\operatorname{tr}_{P_{1}}(q)$.

We show now that $p e_{N}^{P} p=\operatorname{tr}_{P}(p) p q$. It is easy to see that $E_{N}^{P}(m)=$ $[P: N]^{-1} 1_{N}$, hence $E_{N}^{P}(p)=\operatorname{tr}_{P_{1}}(q)^{-1}[P: N]^{-1} 1_{N}=\operatorname{tr}_{P}(p)$. Thus

$$
\begin{aligned}
& \left\|p e_{N}^{P} p-\operatorname{tr}_{P}(p) p q\right\|_{2}^{2} \\
& \quad=\operatorname{tr}_{P_{1}}\left(e_{N}^{P} p e_{N}^{P} p\right)-2 \operatorname{tr}_{P}(p) \operatorname{tr}_{P_{1}}\left(q p e_{N}^{P} p\right)+\operatorname{tr}_{P}(p)^{3} \operatorname{tr}_{P_{1}}(q) \\
& \quad=\operatorname{tr}_{P}(p)^{2}[P: N]^{-1}-2 \operatorname{tr}_{P}(p) \operatorname{tr}_{P_{1}}\left(e_{N}^{P} p\right)+\operatorname{tr}_{P}(p)^{2}[P: N]^{-1}=0 .
\end{aligned}
$$

This implies that $q e_{N_{1}}^{N} q=[P: N] \operatorname{tr}_{P_{1}}(q)^{2} \operatorname{tr}_{P}(p) p q=\operatorname{tr}_{P_{1}}(q) p q$. We have therefore $q P q=\overline{(\operatorname{span} N p N)} q$, in particular $q P q \subset P q$. This allows us to define explicitly the desired conditional expectation. Let $x \in P, y \in P$ with $q x q=y q$, then $y=E(x):=\operatorname{tr}_{P_{1}}(q)^{-1} E_{P}^{P_{1}}(q x q)$. Suppose $x=x^{*} \in P$, then $y q=q x q=(q x q)^{*}=(y q)^{*}=q y$, which shows that $E(x) \in M$ for all $x \in P$. If $x \in M$, then $E(x)=$ $\operatorname{tr}(q)^{-1} E_{P}^{P_{1}}(x q)=\operatorname{tr}(q)^{-1} x E_{P}^{P_{1}}(q)=x$. Furthermore, if we let $\operatorname{tr}_{M}$ 
be the trace on $M$ induced from the trace on $P$, then $\operatorname{tr}_{M}(E(x))=$ $\operatorname{tr}(q)^{-1} \operatorname{tr}_{P_{1}}(q x q)=\operatorname{tr}(q)^{-1} \operatorname{tr}(q) \operatorname{tr}_{P}(x)=\operatorname{tr}_{P}(x)$, i.e. $E$ is indeed the unique trace preserving conditional expectation from $P$ onto $M$ with $q x q=E(x) q$, for all $x \in P$. Hence $q P q=M q=\overline{(\operatorname{span} N p N)} q$, which implies $M=\overline{\operatorname{span} N p N}$. Note that factoriality of $M$ does not follow automatically. Using the Pimsner-Popa estimate ([PiPo1]) it is now easy to see that $\lambda(M, N)^{-1}=\operatorname{tr}_{P}(p)^{-1}(\lambda(M, N)$ denotes the generalized index for non-factors ([PiPo1])) and hence $\lambda(P, M)^{-1}=$ $[P: N] \operatorname{tr}(p)=\operatorname{tr}_{P_{1}}(q)^{-1}$, which says $[P: M]=\operatorname{tr}_{P_{1}}(q)^{-1}$ if $M$ is a factor.

The following corollary gives the desired abstract characterization of Jones projections coming from intermediate subfactors of an irreducible inclusion $N \subset P$.

Corollary 3.3. Let $N \subset P$ be $\mathrm{II}_{1}$ factors with $[P: N]<\infty$ and suppose $N^{\prime} \cap P=\mathbb{C}$. Then $\operatorname{IS}(N, P)$ is precisely the set of Jones projections coming from intermediate subfactors $N \subset M \subset P$ and gives therefore a complete description of the intermediate subfactors of $N \subset P$.

Proof. Apply Proposition 3.1 and Theorem 3.3.

Remark 3.4. (1) Property (3) used in the definition of the set IS $(N, P)$ can be replaced by the following condition: (3)' $q P q \subset$ $P q$. One can then show that (1), (2), (3)' are equivalent to (1), (2) and (3), thus giving an alternative definition of the set $\operatorname{IS}(N, P)$.

(2) Conditions (1)-(3) in the definition of $\operatorname{IS}(N, P)$ do not insure factoriality of the intermediate subalgebra $M$ obtained from $q \in \operatorname{IS}(N, P)$ in general. Of course, if $N \subset P$ is irreducible, then all intermediate subalgebras are factors. Condition (2) will imply factoriality in many cases: if $M$ is not a factor, then $E_{P}^{P_{1}}\left(e_{M}^{P}\right)=E_{M}^{P_{1}}\left(e_{M}^{P}\right)$ is a central element, i.e. of the form $E_{P}^{P_{1}}\left(e_{M}^{P}\right)=\sum_{P_{i=1}}^{r} \alpha_{i} p_{i}$, where $Z(M)=\bigoplus_{i=1}^{r} \mathbb{C} p_{i}, \sum_{i=1}^{r} p_{i}=1_{P}=1_{M}$. Then $E_{P}^{P_{1}}\left(e_{M}^{P}\right)$ will be a scalar iff $\alpha_{i} \equiv$ const., $1 \leq i \leq r$. Whether this happens or not will depend on the traces of the minimal central projections $p_{i}$. For example, if $N \subset M \subset P$ is an intermediate subfactor, then $M \vee\left(M^{\prime} \cap P\right)$ is an intermediate subalgebra, which will not be a factor in general, however the Jones projection corresponding to it may a priori be in IS $(N, P)$. Conversely, if $M$ is an intermediate subalgebra (not necessarily of factor) of $N \subset P$, then it is not clear whether (3) holds in general or not. 
(3) Since we are interested mainly in irreducible subfactors, the corollary gives the desired description of intermediate subfactors in terms of information just coming from $N \subset P$. Furthermore, the Jones projections coming from all intermediate factors are contained in the set $\operatorname{IS}(N, P)$, which will be enough information in many concrete examples.

Subfactors $N \subset P$ with intermediate subfactors as in the corollary are of course easily obtained from group actions, i.e. $N:=R \subset P:=$ $R \rtimes G, G$ a finite (for instance non-simple) group acting properly outer on the hyperfinite $\mathrm{II}_{1}$ factor $R$. We define

Definition 3.5. Let $N \subset P$ be $\mathrm{II}_{1}$ factors, $[P: N]<\infty$, then the inclusion $N \subset P$ is called maximal if there is no subfactor $M$ of $P$ such that $N \subset M \subset P$ other than $N$ and $P$ themselves. Equivalently, $N \subset P, N^{\prime} \cap P=\mathbb{C}$, is maximal iff $\operatorname{IS}(N, P)=\left\{1, e_{N}^{P}\right\}$.

Note that clearly $R \subset R \rtimes G$ is maximal iff the group $G$ has only the trivial group as a subgroup. Since an inclusion $N \subset P$ can only be non-maximal if the index is a product of two indices, we see that all inclusions of index $<4$ and those with index $\in\left(4,8 \cos ^{2} \frac{\pi}{5}\right)$ are certainly maximal. We gave above examples of non-maximal inclusions at index 4 , index $8 \cos ^{2} \frac{\pi}{5}$ and index 6 .

\section{REFERENCES}

[Bi1] D. Bisch, On the existence of central sequences in subfactors, Trans. Amer. Math. Soc., 321 (1990), 117-128.

[Bi2] _ Subfactors arising from groups and their representations, unpublished manuscript.

[B-N] J. Bion-Nadal, An example of a subfactor of the hyperfinite $\mathrm{II}_{1}$ factor whose principal graph invariant is the Coxeter graph $E_{6}$, Current Topics in Operator Algebras, World Scientific Publishing, 1991, pp. 104-113.

[GHJ] F. Goodman, P. de la Harpe and V. F. R. Jones, Coxeter graphs and towers of algebras, MSRI publications 14, Springer, 1989.

[Ha] U. Haagerup, in preparation.

[I1] M. Izumi, Application of fusion rules to classification of subfactors, Publ. RIMS Kyoto Univ., 27 (1991), 953-994.

[I2] _ On flatness of the Coxeter graph $E_{8}$, preprint 1992.

[Jo1] V. F. R. Jones, Index of subfactors, Invent. Math., 72 (1983), 1-25.

[Jo2] _ Hecke algebra representations of braid groups and link polynomials, Ann. of Math., 126 (1987), 335-388.

[Jo3] _ A converse to Ocneanu's theorem, J. Operator Theory, 10 (1983), 61-63.

[Ka] Y. Kawahigashi, On flatness of Ocneanu's connections on the Dynkin diagrams and classification of subfactors, preprint, 1990 . 
[KY] H. Kosaki and S. Yamagami, Irreducible bimodules associated with crossed product algebras, preprint, 1992.

[NT] N. Nakamura and Z. Takeda, On some elementary properties of the crossed products of von Neumann algebras, Proc. Japan Acad., 34 (1958), 489-494.

[Oc1] A. Ocneanu, Quantized group string algebras and Galois theory for operator algebras, in Operator Algebras and Applications 2, London Math. Soc. Lect. Notes Series, 136 (1988), 119-172.

[Oc2] _ Quantum symmetry, differential geometry of finite graphs and classification of subfactors, University of Tokyo Seminar Notes 45, 1991 (Notes recorded by $Y$. Kawahigashi).

[PiPo1] M. Pimsner and S. Popa, Entropy and index of subfactors, Ann. Scient. Ec. Norm. Sup., 19 (1986), 57-106.

[PiPo2] _ Iterating the basic construction, Trans. Amer. Math. Soc., 310 (1988), 127-133.

[Po1] S. Popa, Sousfacteurs, actions des groupes et cohomologie, C. R. Acad. Sci. Paris, Série I, 309 (1989), 771-776.

[Po2] _ Classification of subfactors: reduction to commuting squares, Invent. Math., 101 (1990), 19-43.

[Po3] _ Sur la classification des sous-facteurs d'indice fini du facteur hyperfini, C. R. Acad. Sci. Paris, Série I, 311 (1990), 95-100.

[Po4] _ Classification of amenable subfactors and their automorphisms, preprint, 1991.

[SV] V. S. Sunder and A. K. Vijayaran, On the non-occurrence of the Coxeter graphs $E_{7}, D_{2 n+1}$ as principal graphs of an inclusion of $\mathrm{II}_{1}$ factors, preprint, 1991.

[Su] N. Suzuki, Crossed products of rings of operators, Tohoku Math. J., 11 (1959), 113-124.

[We1] H. Wenzl, Hecke algebras of type $A$ and subfactors, Invent. Math., 92 (1988), 345-383.

[We2] _ Quantum groups and subfactors of type B, C and D, Comm. Math. Phys., 133 (1990), 383-432.

Received April 15, 1992 and in revised form August 16, 1992. Supported by an MSRI postdoctoral fellowship through NSF grant DMS 8505550.

Mathematical Sciences Research Institute

1000 CENTENNIAL DRIVE

BERKELEY, CA 94720

Current address: Department of Mathematics

University of California, Berkeley

Berkeley, CA 94720 


\section{CONTENTS}

D. Bisch, A note on intermediate subfactors , . , . . . . . . . . . . . . . . . . 201

M. J. Carro and J. Soria, Tent spaces over general approach regions and pointwise

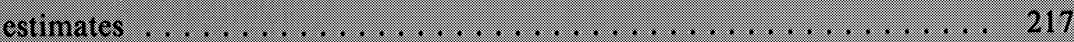

J. A. Charris, M. E. H. Ismail, and S. Monsalve, On sieved orthogonal polynomials X: general blocks of recurrence relations . . . . . . . . . . . . . . . . 237

K. S. Chou and T. Y. H. Wan, Asymptotic radial symmetry for solutions of $\Delta u+$ $e^{\prime \prime}=0$ in a punctured disc . . . . . . . . . . . . . . . . . . . . . . . 269

M. E. Fogel, Knots with algebraic unknotting number one . . . . . . . . . . . . . 277

K. N. Jones, The structure of closed nonpositively curved Euclidean cone 3-manifolds . . . . . . . . . . . . . . . . . . . . . . . . . . . . . . . . 297

M. Kaneda, On the Frobenius morphism of flag schemes . . . . . . . . . . 315

H. T. Kaptanoğlu, Möbius-invariant Hilbert spaces in polydiscs . . . . . . . . . 337

K. Kuribayashi, The cohomology ring of the spaces of loops on Lie groups and homogeneous spaces ............................ 361

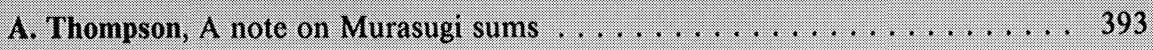




\title{
PACIFIC JOURNAL OF MATHEMATICS
}

\author{
Volume $163 \quad$ No. $2 \quad$ April 1994
}

A note on intermediate subfactors

201

DIETMAR BISCH

Tent spaces over general approach regions and pointwise estimates

217

MARÍA J. CARRO and JAVIER SORIA

On sieved orthogonal polynomials. X. General blocks of recurrence

237 relations

JAIro A. Charris, Mourad ISMAIL and SERGio Monsalve

Asymptotic radial symmetry for solutions of $\Delta u+e^{u}=0$ in a punctured disc

Kai Seng (Kaising) Chou (Tso) and Tom YaU-Heng Wan

Knots with algebraic unknotting number one

MicAh Elton Fogel

The structure of closed non-positively curved Euclidean cone

3-manifolds

KERRY NELSON JONES

On the Frobenius morphism of flag schemes

MASAHARU KANEDA

Möbius-invariant Hilbert spaces in polydiscs

H. TuRgay KaptanOGLU

The cohomology ring of the spaces of loops on Lie groups and homogeneous spaces

KATSUHIKO KURIBAYASHI

A note on Murasugi sums

ABIGAIL A. THOMPSON 